\title{
Comparative study on the diagnostic values of different ultrasound technologies for malignant thyroid nodules
}

\author{
XIAO-ZHU JIN ${ }^{1 *}$, WEI-WEI LU ${ }^{2 *}$, HAI-FENG ZHANG ${ }^{3}$, YUAN-YUAN YAN $^{1}$ and XIAO-LE GU ${ }^{1}$ \\ ${ }^{1}$ Department of Ultrasonography, Zhengzhou Central Hospital Affiliated to Zhengzhou University, \\ Zhengzhou, Henan 450000; ${ }^{2}$ Department of Ultrasonography, The First People's Hospital of Shangqiu, \\ Shangqiu, Henan 476000; ${ }^{3}$ Department of Radiology, Zhengzhou Central Hospital Affiliated to \\ Zhengzhou University, Zhengzhou, Henan 450000, P.R. China
}

Received April 11, 2017; Accepted January 25, 2018

DOI: $10.3892 / \mathrm{ol} .2018 .8706$

\begin{abstract}
The present study compared and analyzed the diagnostic values of B-mode ultrasound and elasticity imaging technology for the identification of benign and malignant thyroid nodules. Ninety-four patients who were diagnosed with thyroid nodules by ultrasound were chosen. All patients were checked with B-mode ultrasound and ultrasound elasticity imaging technology before surgeries. Further, the post-operative outcomes were compared with operation pathology. The pathological examination results were taken as the gold standard. The accuracy, sensitivity, specificity, positive predictive value and negative predictive values of combined ultrasound diagnosis were all obviously higher than those by the B-mode ultrasound and the ultrasound elastography method $(\mathrm{P}<0.05)$. In conclusion, as a new technology, ultrasound elasticity imaging technology has relatively high diagnositic value in identifying benign and malignant thyroid nodules. With its own advantages and limitations, the ultrasound elasticity imaging technology could effectively complement B-mode ultrasound, and improve the sensitivity, specificity and accuracy of ultrasound diagnosis for thyroid nodules.
\end{abstract}

\section{Introduction}

Thyroid nodule refers to a mass of abnormally proliferating cells with abnormal local hardness in thyroid (1). At present, ultrasonography of thyroid nodules mainly relies on B-mode ultrasound diagnosis. However, the diagnostic sensitivity and

Correspondence to: Dr Xiao-Le Gu, Department of Ultrasonography, Zhengzhou Central Hospital Affiliated to Zhengzhou University, 195 Tongbai Road, Zhongyuan, Zhengzhou, Henan 450000, P.R. China

E-mail: o52341283huang@163.com

*Contributed equally

Key words: B-mode ultrasound, elasticity imaging techniques, thyroid nodule, benign, malignant specificity by B-mode ultrasound is not high $(2,3)$. Ultrasound elasticity imaging, as a new technology developed this year, can determine the mechanical characteristics of tissues in vitro (4). The application value of ultrasound elasticity imaging technology in breast cancer has been confirmed, but its applications in the diagnosis of benign and malignant thyroid nodules are relatively few. The present study compared diagnostic values of B-mode ultrasound and elasticity imaging for the identification of benign as well as malignant thyroid nodules. This modality helps to improve the diagnosis rate of benign and malignant thyroid nodules by ultrasound technology.

\section{Materials and methods}

General materials. Ninety-four confirmed cases (from January, 2011 to January, 2015) of thyroid nodules by ultrasound in Zhengzhou Central Hospital Affiliated to Zhengzhou University (Zhengzhou, China) were chosen for the study. There were 46 male and 48 female cases. The age of the patient ranged from 22-72 years and the average age was $43.5 \pm 4.5$ years.

Detection methods. Siemens Acuson Antares 5.0 Color Doppler Ultrasonic Diagnosis Apparatus (Siemens, Erlangen, Germany) and VF13-5 frequency conversion probe were adopted as the detection instrument. All study subjects were examined by B-mode ultrasound and ultrasound elastography. Patients were kept in supine position and were first given routine vertical and horizontal section scanning of the neck. Nodule forms, sizes, boundaries, internal echo, existence of calcification, surrounding acoustic halo, blood flow conditions and cervical lymph nodes were observed by two-dimensional ultrasound. Subjects were then analyzed by elasticity imaging mode. We tried to make the probe cling to the neck skin during the operation. It was better to make the nodule at the center of sample frame. The size of sample frame was supposed to be adjusted to about twice the size of the lesions. The probe was held by hand and made to slightly vibrate at masses at middle speed. We saved the picture, when stress figure on the device monitor was between 3-4 grades. The color changes of elasticity picture in the sample frame were observed (5). 
Table I. The classification results and pathological diagnosis of thyroid nodule ultrasound elasticity.

\begin{tabular}{|c|c|c|c|c|c|}
\hline \multirow[b]{2}{*}{$\begin{array}{l}\text { Ultrasound } \\
\text { elasticity grade }\end{array}$} & \multicolumn{2}{|c|}{ Benign } & \multicolumn{3}{|c|}{ Malignant } \\
\hline & $\begin{array}{l}\text { Nodular } \\
\text { goiter }\end{array}$ & $\begin{array}{l}\text { Thyroid } \\
\text { adenoma }\end{array}$ & $\begin{array}{l}\text { Papillary } \\
\text { carcinoma }\end{array}$ & $\begin{array}{l}\text { Follicular thyroid } \\
\text { carcinoma }\end{array}$ & $\begin{array}{c}\text { Non-hodgkin's } \\
\text { lymphoma }\end{array}$ \\
\hline Grade 0 & 7 & 6 & 0 & 0 & 0 \\
\hline Grade I & 0 & 9 & 2 & 0 & 0 \\
\hline Grade II & 10 & 12 & 1 & 0 & 0 \\
\hline Grade III & 8 & 17 & 7 & 1 & 0 \\
\hline Grade IV & 1 & 2 & 9 & 1 & 1 \\
\hline Total & 26 & 46 & 19 & 2 & 1 \\
\hline
\end{tabular}
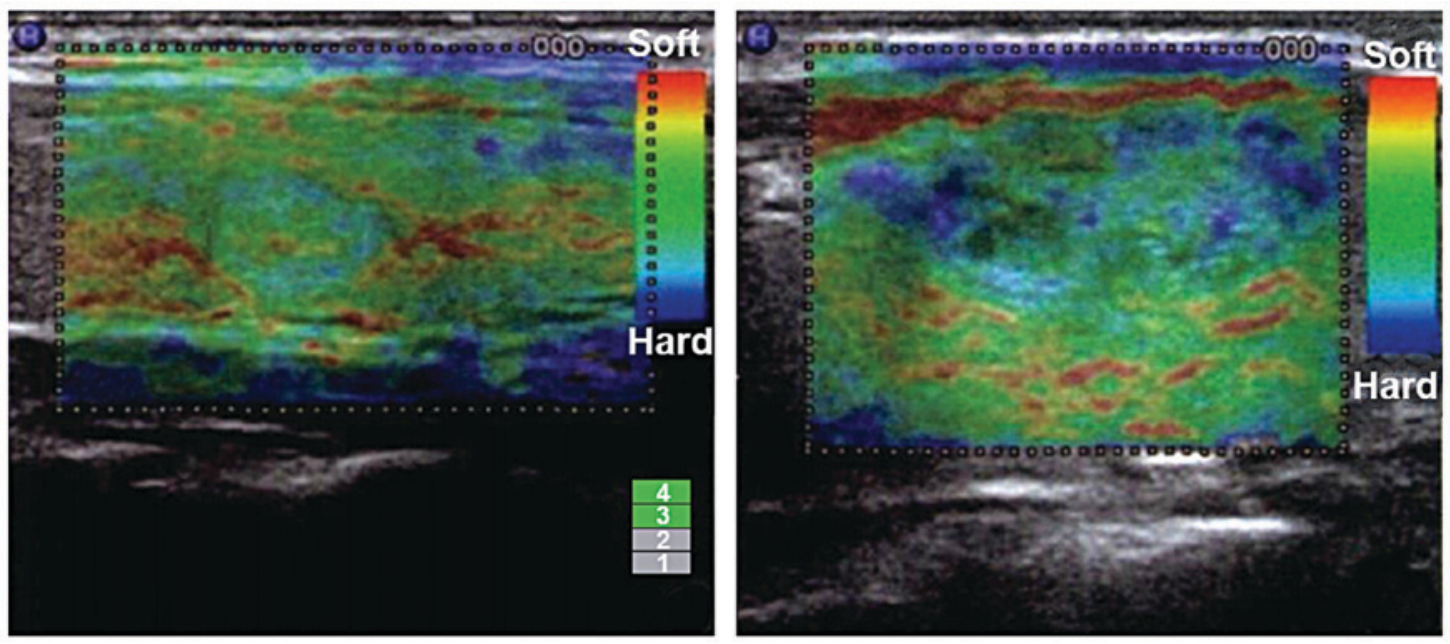

Figure 1. Elasticity ultrasound imaging of the thyroid.

Diagnostic criteria of B-mode ultrasound. The features of benign thyroid nodules were regular morphology, clear boundary, $<1$ aspect ratio, inside equal echo, high echo, with or without an echo, coarse calcifications, no rear echo reduction, no or a little blood flow. The resistive index of blood flow was $<0.7$. The features of malignant thyroid nodules included irregular forms, unclear boundary, the aspect ratio was $\geq 1$, inside low echo, micro-calcification, rear echo reduction, rich blood flow. The resistive index of blood flow was $\geq 0.7$.

Diagnostic criteria of ultrasound elastography. We adopted the hardness-scoring standard recommended by the Hitachi Co. and took the 5 fractions method according to different nodule colors. One point, the cystic components predominated in nodules manifested by blue-green colors; 2 points, nodules and surrounding tissues manifested by evenly green color; 3 points, $>50 \%$ and $<90 \%$ nodules manifested by green color; 4 points, $>50 \%$ and $<90 \%$ nodules manifested by blue color; 5 points, $>90 \%$ nodules manifested by blue color; $1-3$ points indicated benign thyroid nodules, and 4-5 points indicated malignant thyroid nodules (6). Fig. 1 shows the elasticity ultrasound imaging of the thyroid.

Statistical analysis. Data were analyzed and processed by SPSS 18.0 (SPSS, Inc., Chicago, IL, USA). Enumeration data were examined by $\chi^{2}$ test. $\mathrm{P}<0.05$ was considered to be statistically significant.

\section{Results}

Results of pathological findings. Among 94 thyroid nodules, there were 22 malignant nodules, including 2 cases of follicular thyroid carcinoma, 9 cases of papillary carcinoma and 1 case of metastatic carcinoma. Further, there were 72 benign nodules, including 26 cases of goiter nodules and 46 cases of thyroid adenoma (Table I).

The B-mode ultrasound evaluation of benign and malignant thyroid nodules. The identification of benign and malignant thyroid nodules by the B-mode ultrasound showed $84.04 \%$ (79/94) accuracy, $77.27 \%$ (17/22) sensitivity, $86.11 \%$ (62/72) specificity, $62.96 \%$ (17/27) positive predictive value and $92.53 \%$ (62/67) negative predictive value. On the other hand, the ultrasound elastography showed $86.17(81 / 94)$ accuracy, 81.82\% (18/22) sensitivity, 87.50\% (63/72) specificity, $66.67 \%$ (18/27) positive predictive value and 94.02\% (63/67) negative predictive value. Further the identification of benign and malignant thyroid nodules by the combined ultrasound diagnosis showed $96.81 \%$ (91/94) accuracy, 95.45\% (21/22) sensitivity, $97.22 \%$ (70/72) specificity, $91.30 \%$ (21/23) positive 


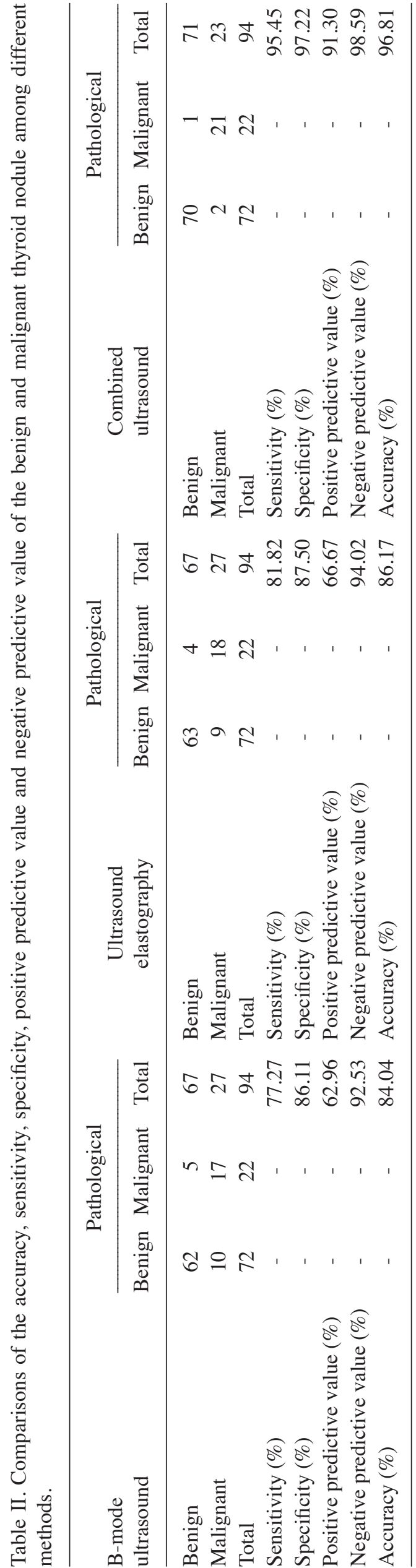

predictive value and $98.59 \%$ (70/71) negative predictive value. The accuracy, sensitivity, specificity, positive predictive value and negative predictive values of combined ultrasound diagnosis were all obviously higher than those by the B-mode ultrasound method and the ultrasound elastography method $(\mathrm{P}<0.05)$. There were no significant differences between results of the B-mode ultrasound method and the ultrasound elastography method ( $\mathrm{P}>0.05)$ (Table II).

\section{Discussion}

China has listed the thyroid nodule as a health examination item, as the detection rates are on the rise continuously. Five to $10 \%$ of these thyroid nodules are malignant nodules (7). The early diagnosis would contribute to increasing the cure rate, prolonging the survival time and improving the survival quality of patients. Thyroid nodules are relatively occult at early stage. Therefore, it is difficult to find the nodule completely by preliminary diagnosis. The B-mode ultrasound diagnosis could not effectively identify the nodule either, which is easy to be confused with the thyroid tissue. For a long time, ultrasound has been preferred for diagnosis of benign and malignant thyroid nodules. Thus, the B-mode ultrasound detection is not able to identify all types of benign or malignant thyroid nodules (2).

The prime research target in imaging currently is to improve the detection rate and reduce the omission diagnostic rate of malignant thyroid nodules. The real-time ultrasound elastography is a newly developed ultrasound technology (8). This technology reflects the hardness of monitored tissues mainly by detecting tissue distortion degree by applying external pressure. Images are developed in accordance with hardness differences among tissues. Ultrasound elastography could provide information on elasticity, which is the basic mechanic property so as to provide a new way of identifying benign and malignant thyroid nodules. This study evaluated advantages and disadvantages of B-mode ultrasound and ultrasound elastography in identifying benign and malignant thyroid nodules. We combined the B-mode ultrasound with ultrasound elastography for diagnosis, and found that identification of benign and malignant thyroid nodules by the combined ultrasound showed good results. The accuracy was greatly improved which indicated that the elasticity imaging technology could raise the accuracy of identifying benign and malignant thyroid nodule by B-mode ultrasound.

In the present study, we found that the accuracy, sensitivity, specificity and positive predicative value of combined ultrasound were all better than those of B-mode ultrasound and ultrasound elastography. Results above showed that there is no significant difference between the diagnostic accuracy of thyroid nodules by B-mode ultrasound and ultrasound elastography. Perhaps it is because the elasticity imaging technology could provide information related to tissue elasticity and is affected by many factors. Goertz et al (9) have compared the results of B-mode ultrasound and the elasticity imaging technology and found the latter showed no advantages over the former. However, the accuracy between the two technologies in diagnosis of thyroid nodules revealed no statistical significance. Ultrasound elasticity imaging is a technology aiming at tumor examinations and gives relatively ideal imaging effect of 
tumors or diffused diseases. At present, most clinical studies suggested that B-mode ultrasound is better in the aspects of accuracy, sensitivity and specificity. However, ultrasound elastography is limited in its improvement. Therefore, in recent years, a number of literature data have focused on the diagnosis effects of B-mode ultrasound detection method combined with the elasticity imaging technology. All research indicated that combined detection has greatly increased the diagnosis sensitivity, specificity and positive predicative values, which in turn conform to our research results (10-12).

Ultrasound elasticity imaging technology identified the hardness, elasticity and pathological characters of lesion tissues. So it could serve as the basis of pathological diagnosis. Its application in pathological diagnosis of breast diseases is relatively good. The identification accuracy of benign and malignant tissues reached $89.6-91.3 \%$ which is a relatively good directive significance for clinical diagnosis (10). However, thyroid nodule tissues are different from breast tissues as the density difference among benign and malignant nodules is smaller than that of breast tumors. So, the diagnosis accuracy of benign and malignant tissues is reduced. It is still a kind of significant iconography detection methods with wide application. There are many reliable reports on its individual application. Also, there are some controversial reports (11). In terms of the diagnosis of benign and malignant thyroid nodules, some scholars suggested that the elasticity imaging technology could not be used as an independent diagnosis method (5). Ultrasound elasticity imaging technology has its own advantages of identifying lesion nature. Ultrasound elasticity imaging could locate tumors and reveal the relationships between tumors and surrounding tissues. In recent years, the application of real-time tissue elastography (13-15) technology allowed recording of real-time color images. Judgment of the nature of the tumor could be more exact based on echo signal movement. The diagnosis effect would be further improved in the combination of ultrasound elasticity imaging with routine inspection. Therefore, it is more appropriate to take ultrasound elastography as an assisting detection method for B-mode ultrasound examination (16-18).

To conclude, ultrasound elasticity imaging technology, as a new technology, has relatively high diagnotic value for identifying benign and malignant thyroid nodules. The ultrasound elasticity imaging technology could effectively complement B-mode ultrasound diagnosis, and improve the sensitivity, specificity and accuracy of ultrasound diagnosis of thyroid carcinoma.

\section{Acknowledgements}

Not applicable.

\section{Funding}

No funding was received.

\section{Availability of data and materials}

The datasets used and/or analyzed during the current study are available from the corresponding author on reasonable request.

\section{Authors' contributions}

XZJ contributed significantly to writing the manuscript and exam of patients by ultrasound. WWL and HFZ helped with B-mode ultrasound. YYY and XLG analyzed and interpreted ultrasound elastography result. All authors read and approved the final manuscript.

\section{Ethics approval and consent to participate}

The study was approved by the Ethics Committee of Zhengzhou Central Hospital Affiliated tot Zhengzhou University (Zhengzhou, China) and informed consents were signed by the patients and/or guardians.

\section{Consent for publication}

Not applicable.

\section{Competing interests}

The authors declare that they have no competing interests.

\section{References}

1. Wong CK and Wheeler MH: Thyroid nodules: Rational management. World J Surg 24: 934-941, 2000.

2. Dudea SM and Botar-Jid C: Ultrasound elastography in thyroid disease. Med Ultrason 17: 74-96, 2015.

3. Frates MC, Benson CB, Charboneau JW, Cibas ES, Clark OH, Coleman BG, Cronan JJ, Doubilet PM, Evans DB, Goellner JR, et al; Society of Radiologists in Ultrasound: Management of thyroid nodules detected at US: Society of Radiologists in Ultrasound consensus conference statement. Radiology 237: 794-800, 2005.

4. Rago T, Santini F, Scutari M,Pinchera A and Vitti P: Elastography: New developments in ultrasound for predicting malignancy in thyroid nodules. J Clin Endocrinol Metab 92: 2917-2922, 2007.

5. Jiang Y, Li GY, Qian LX, Hu XD, Liu D, Liang S and Cao Y: Characterization of the nonlinear elastic properties of soft tissues using the supersonic shear imaging (SSI) technique: Inverse method, ex vivo and in vivo experiments. Med Image Anal 20: 97-111, 2015.

6. Li T, Zhou P, Zhang X, Ding M, Yuchi M and Li Y: Diagnosis of thyroid nodules using virtual touch tissue quantification value and anteroposterior/transverse diameter ratio. Ultrasound Med Biol 41: 384-392, 2015.

7. Mehrmohammadi M, Song P, Meixner DD, Fazzio RT, Chen S, Greenleaf JF, Fatemi M and Alizad A: Comb-push ultrasound shear elastography (CUSE) for evaluation of thyroid nodules: Preliminary in vivo results. IEEE Trans Med Imaging 34: 97-106, 2015.

8. Ophir J, Céspedes I, Ponnekanti H, Yazdi Y and Li X: Elastography: A quantitative method for imaging the elasticity of biological tissues. Ultrason Imaging 13: 111-134, 1991.

9. Goertz RS: Ultrasound elastography. Radiologe 55: 949-955, 2015 (In German).

10. Yoon JH, Yoo J, Kim EK, Moon HJ, Lee HS, Seo JY, Park HY, Park WJ and Kwak JY: Real-time elastography in the evaluation of diffuse thyroid disease: A study based on elastography histogram parameters. Ultrasound Med Biol 40: 2012-2019, 2014.

11. Zhuo J, Ma Z, Fu WJ and Liu SP: Differentiation of benign from malignant thyroid nodules with acoustic radiation force impulse technique. Br J Radiol 87: 20130263, 2014.

12. Sun J, Cai J and Wang X: Real-time ultrasound elastography for differentiation of benign and malignant thyroid nodules: A meta-analysis. J Ultrasound Med 33: 495-502, 2014.

13. Udelsman R and Zhang Y: The epidemic of thyroid cancer in the United States: The role of endocrinologists and ultrasounds. Thyroid 24: 472-479, 2014.

14. Wells PN and Liang HD: Medical ultrasound: Imaging of soft tissue strain and elasticity. J R Soc Interface 8: 1521-1549, 2011. 
15. Giovanella L, Clark PM, Chiovato L, Duntas L, Elisei R, Feldt-Rasmussen U, Leenhardt L, Luster M, Schalin-Jäntti C, Schott M, et al: Thyroglobulin measurement using highly sensitive assays in patients with differentiated thyroid cancer: A clinical position paper. Eur J Endocrinol 171: R33-R46, 2014.

16. Remonti LR, Kramer CK, Leitão CB, Pinto LC and Gross JL: Thyroid ultrasound features and risk of carcinoma: A systematic review and meta-analysis of observational studies. Thyroid 25 : 538-550, 2015.

17. Doherty JR, Trahey GE, Nightingale KR and Palmeri ML: Acoustic radiation force elasticity imaging in diagnostic ultrasound. IEEE Trans Ultrason Ferroelectr Freq Control 60: 685-701, 2013.
18. Andrioli M and Persani L: Elastographic techniques of thyroid gland: Current status. Endocrine 46: 455-461, 2014.

c) (i) $\Theta$ This work is licensed under a Creative Commons Attribution-NonCommercial-NoDerivatives 4.0 International (CC BY-NC-ND 4.0) License. 